\title{
Comparison of Volatile Components between Raw and Vinegar Baked Radix Bupleuri by GC-MS Based Metabolic Fingerprinting Approach
}

\author{
Jie Xing, ${ }^{1,2}$ Hui-Min Sun, ${ }^{1,2}$ Zhen-Yu Li, ${ }^{1}$ and Xue-Mei Qin ${ }^{1}$ \\ ${ }^{1}$ Modern Research Center for Traditional Chinese Medicine of Shanxi University, No. 92, Wucheng Road, \\ Taiyuan, Shanxi 030006, China \\ ${ }^{2}$ College of Chemistry and Chemical Engineering of Shanxi University, No. 92, Wucheng Road, Taiyuan, Shanxi 030006, China
}

Correspondence should be addressed to Zhen-Yu Li; lizhenyu@sxu.edu.cn and Xue-Mei Qin; qinxm@sxu.edu.cn

Received 3 April 2015; Revised 24 June 2015; Accepted 2 July 2015

Academic Editor: Hyunsu Bae

Copyright (C) 2015 Jie Xing et al. This is an open access article distributed under the Creative Commons Attribution License, which permits unrestricted use, distribution, and reproduction in any medium, provided the original work is properly cited.

\begin{abstract}
Radix Bupleuri (RB), also named Chaihu in Chinese, is a commonly used herbal drug in traditional Chinese medicine (TCM), and the processing of RB with vinegar to prepare vinegar-baked Radix Bupleuri (VBRB) has a long history in the clinic of TCM. In the present study, GC-MS coupled with multivariate data analysis was applied to compare the volatile components between crude and two vinegar processed RBs. After vinegar baking, the oil yields were decreased significantly, and the chemical compositions were also changed greatly. The chemical changes included the disappearance or appearance, as well as the content increase or decrease of some volatile compounds. The oil yields of two different VBRBs showed no significant difference but differed markedly in their chemical compositions, suggesting that the type of vinegar exerted great impacts on the vinegar-baking process. Thus, the effect of different vinegars on processing should be further investigated to ensure the therapeutic effect and safety of VBRB in clinic.
\end{abstract}

\section{Introduction}

Radix Bupleuri ("chaihu" in Chinese, short for RB), the dry root of Bupleurum chinense DC. or B. scorzonerifolium Willd. (Apiaceae), is a commonly used herbal drug in traditional Chinese medicine (TCM) and plays an important role in the treatment of many diseases such as influenza, fever, malaria, hepatitis, jaundice, nephritis, dizziness, lung diseases, cancer, and menstrual disorders in China, Japan, and other Asian countries [1-4]. Chemical investigation of RB revealed the presence of saikosaponins, volatile oils, flavonoids, coumarins, fatty acids, steroids, polysaccharides, and polyacetylenes $[5,6]$.

Processing of herbal drugs has been a part of the heritage of Chinese medicine for thousands of years; it plays an important role in disease prevention and control for the Chinese people and ensures the safe and effective clinical treatment of TCM [7]. When RB is mixed with vinegar and then baked to dry, it is changed to vinegar-baked Radix Bupleuri (VBRB). The pharmacological effects and components in the drug will change a little bit due to the vinegar-baking procedure. The bile secreting and hepatoprotective effects are enhanced and it is quite effective in curing liver related diseases such as jaundice, hepatitis, cirrhosis, and liver cancer [8-11]. Previous reports showed that the contents of saikosaponin b1 and saikosaponin b2 were increased, while the saikosaponin a, saikosaponin $\mathrm{c}$, and saikosaponin d were decreased [12, 13] after the vinegar-baking process. Previous investigations have demonstrated that the volatile oil of $\mathrm{RB}$ showed the effects of anti-influenza, antipyretic, anti-inflammation, and analgesia $[14,15]$. After being processed with vinegar, both the yields $[16,17]$ and compositions [18] of volatile oil in Radix Bupleuri could be changed. In addition, according to Chinese Pharmacopoeia, RB should be baked with rice vinegar to give VBRB. However, there are many kinds of vinegars present in the Chinese market, and most of them 
were used in the vinegar-baking process of herbal drugs. The influence of different vinegars on the volatile compounds in VBRB remains unknown.

Steam distillation and solvent extraction methods combined with gas chromatography (GC) or gas chromatography-mass spectrometry (GC/MS) are used as the routine methods for the analysis of the volatile oils of TCMs. And GCMS is one of the most robust methodologies widely applied in volatile metabolite analysis because of its high sensitivity, peak resolution, and reproducibility $[19,20]$. Recently, GCMS-based global metabolic profiling, coupled with multivariate analysis, has been successfully applied to quality assessment of volatile compounds in herbal drugs, such as agarwood [21], Cassia [22], Ginseng [23], and Atractylodis Macrocephalae Rhizoma [24].

In this study, two different vinegars were used to prepare VBRB. And the aim of the present study was to investigate and compare the chemical differences of the volatile oils between crude and vinegar-processed RB samples by GC-MS coupled with multivariate data analysis. Two different VBRBs were also compared to elucidate the vinegar type on vinegarbaking process of RB.

\section{Materials and Methods}

2.1. Plant Materials. The Radix Bupleuri was purchased from Shanxi Weikangtang Chinese herbal pieces company and authenticated by Professor Xue-Mei Qin as B. chinense DC. A voucher specimen (lot number $\mathrm{CH}-46$ ) was deposited at Modern Research Center for Traditional Chinese Medicine of Shanxi University.

2.2. Solvents and Chemicals. Analytical grade n-hexane was purchased from Beijing Chemical works (Beijing, China) and n-tetracosane (purity $>98 \%$ ) which used as an internal quality standard for GC-MS analysis was bought from Johnson Matthey Company (Shanghai, China). Bran vinegar was bought from Tongwanzhenji Food Company (Hebei, China) and Shanxi vinegar was from Shanxi Donghu Vinegar Group (Shanxi, China).

2.3. VBRB Preparation. According to Chinese Pharmacopoeia, the crude RB (100 g) was incubated with Shanxi vinegar or rice vinegar $(20 \mathrm{~g})$, respectively. Then the material was dried by stir-firing to obtain S-VBRB (by Shanxi vinegar) or R-VBRB (by rice vinegar) after vinegar was totally absorbed into raw RB. Six different batches of S-VBRB and $\mathrm{R}-\mathrm{VBRB}$ were prepared for each kind of vinegars.

\subsection{Extract of Volatile Oil and GC-MS Analysis}

2.4.1. Extraction of Volatile Oil. Steam distillation, a typical extraction method for volatile oils, was chosen according to the Chinese Pharmacopoeia [1]. The dried powder (30 g) was accurately weighed and transferred to a $500 \mathrm{~mL}$ roundbottomed flask soaked in $240 \mathrm{~mL}$ of water for $2 \mathrm{~h}$. Water was added from the top of the volatile oil determination apparatus until the water spilled onto the round-bottomed flask and $2 \mathrm{~mL}$ of $\mathrm{n}$-hexane was added to the water layer. Then the essential oils were extracted by water distillation for $6 \mathrm{~h}$. Volatile oil was separated from the water layer and leached into the n-hexane layer, and then the n-hexane layer was dried over anhydrous sodium sulfate $\left(\mathrm{Na}_{2} \mathrm{SO}_{4}\right)$ and weighted. The samples were stored at $4^{\circ} \mathrm{C}$ in the refrigerator before GC-MS analysis. All samples were prepared in sextuplicate.

2.4.2. GC-MS Analysis Parameters. GC-MS analysis was performed using a Polaris Q ion trap mass spectrometer (Thermo Fisher Scientific Inc., USA). Chromatography was performed on a DB-5MS capillary column $(30 \mathrm{~m} \times 250 \mu \mathrm{m}$ i.d., $0.25 \mu \mathrm{m}$ film thickness; $5 \%$ diphenyl cross-linked $95 \%$ dimethylpolysiloxane; Agilent J\&W Scientific, Folsom, CA). Helium carrier gas was used at a constant flow rate of $1 \mathrm{~mL} \cdot \mathrm{min}^{-1}$. Approximately $1.0 \mu \mathrm{L}$ of samples was injected at a constant temperature of $250^{\circ} \mathrm{C}$ in splitless mode. Initial temperature was set to $50^{\circ} \mathrm{C}$ and held for $1 \mathrm{~min}$, followed by a ramp to $100^{\circ} \mathrm{C}$ at $10^{\circ} \mathrm{C} \cdot \mathrm{min}^{-1}$ and held for $2 \mathrm{~min}$ and then to $180^{\circ} \mathrm{C}$ at $3^{\circ} \mathrm{C} \cdot \mathrm{min}^{-1}$, and then rose to $220^{\circ} \mathrm{C}$ at $15^{\circ} \mathrm{C} \cdot \mathrm{min}^{-1}$ and maintained $1 \mathrm{~min}$ and post-run temperature to $300^{\circ} \mathrm{C}$ for $5 \mathrm{~min}$. The solvent delay was set as $5 \mathrm{~min}$. The interface and source temperatures were set at $280^{\circ} \mathrm{C}$ and $200^{\circ} \mathrm{C}$, respectively. MS detection was implemented with electron ionization (electron energy of $70 \mathrm{eV}$ ) and full scan mode $(m / z 50-650)$.

2.4.3. Compound Identification. The components eluting within the total ion chromatogram were extracted in AMDIS, matrix interference was then resolved, and overlapping components were removed. Then the compounds were positively identified using the National Institute of Standards and Technology (NIST) 05L Mass Spectra Database containing about 107,000 compounds, as well as comparison with the literatures $[18,25,26]$. The semiquantitative analysis of volatile compounds was performed by comparing their peak areas to that of the internal standard compound on the GCMS total ion chromatogram. The percentage compositions of compounds were calculated by area normalization method.

2.4.4. Data Analysis. To assess difference (or similarity) between RB and VBRBs, principal component analysis (PCA) was applied to relative peak area values of volatiles obtained on the GC-MS total ion chromatograms using SIMCAP13.0 (Umetrics, Umeå, Sweden) to clarify the relationship between the RB and VBRBs. In addition, hierarchical heat map clustering analysis was performed with MetaboAnalyst (http://www.metaboanalyst.ca/). The significance level was set at $p<0.05$ for all tests by SPSS 16.0.

\section{Results and Discussion}

3.1. Determination of Volatile Oil Yields. The oil yields were $0.72 \pm 0.05,0.58 \pm 0.09$, and $0.53 \pm 0.05(\mathrm{mg} / \mathrm{g})$ for RB, S$\mathrm{VBRB}$, and R-VBRB, respectively. And there was significant difference between the oil yields of crude and processed RBs $(p<0.05)$, while the S-VBRB and R-VBRB showed no significant difference $(p=0.18)$. 

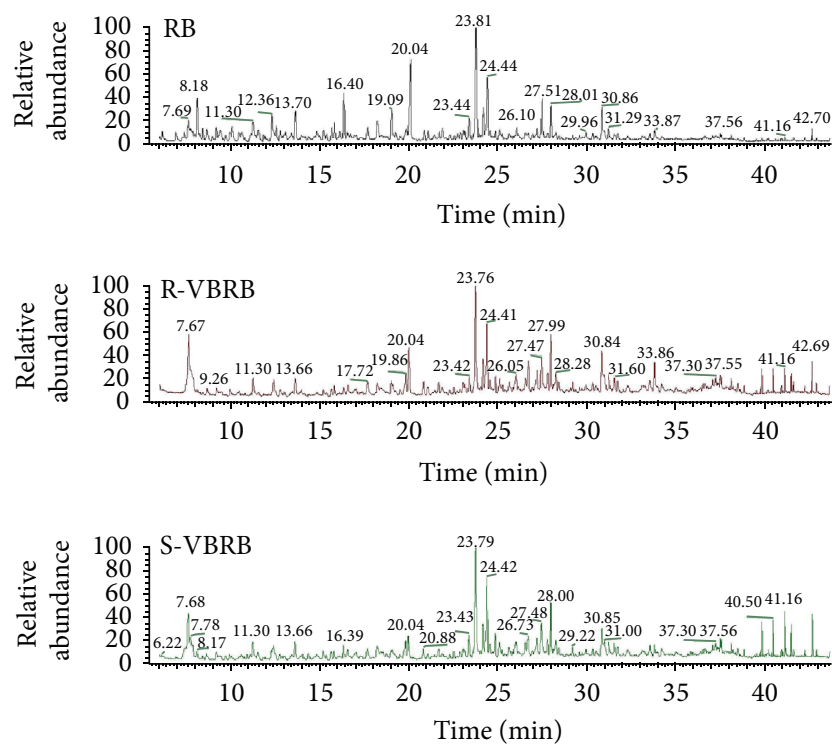

FIGURE 1: GC-MS chromatograms of raw and processed Radix Bupleuri.

3.2. Volatile Compounds Identification. All samples were analyzed by GC-MS, and the TIC chromatograms are shown in Figure 1. A total of 59 compounds were identified in crude and processed RB samples, which amounted for about $75 \%$ of the total essential oil, including 15 monoterpenes, 8 sesquiterpenes, 10 aldehydes, 7 phenols (including their esters and ethers), 4 alkane, 3 alcohols, 6 fatty acids, and 6 miscellaneous compounds (Table 1). In the total essential oil, $\beta$-pinene, 1-methyl-2-isopropyl benzene, 1-methyl-4-(1methylethyl)-cyclohexadiene, 1-methyl-4-isopropyl benzene, 1-methyl-4-(1-methyethylidene)-cyclohexene, verbenol, 2-(1, 1-dimethylethyl)-phenol, 1-isopropyl-2-methoxy-4-toluene, 4-dimethyl-3-cyclohexene-1-acetaldehyde, eucalyptol, menthol, methychavicol, thymol, 2-methyl-5-(1-methylethyl)phenol, and moslene as the monoterpenes and pentanal, n-hexanal, dodecanal, benzaldehyde, n-nonaldehyde, (E)2-octenal, (E)-2,4-nonadienal, (E,E)-2,4-sebacic olefin aldehyde, (Z)-2-decenal, and capraldehyde as the aldehydes were determined as the main components.

In addition, 6 compounds ( $\beta$-pinene, 1-methyl-4-(1methylethyl)-cyclohexadiene, 1,2-cyclooctene oxide, n-nonaldehyde, verbenol, and 2-decenal) found in crude RB samples were disappeared in processed RB samples, while 5 compounds (2,4-dimethoxytoluene, 2-nonyl acetylene, $\alpha$-cubebene, 6-methyl-2-(4-methylphenyl)-5-heptylene, and nerolidol) were newly generated and identified in VBRBs.

3.3. Chemical Difference of Raw and Vinegar-Baked RBs by Multivariate Analysis. Since the oils yields of RB decreased significantly after the vinegar-baking process, the change of chemical compositions between raw and processed RBs should be further investigated. Thus, all the GC-MS data (134 peaks) were subjected to PCA analysis to visualize the chemical difference between the raw and vinegar-baked RBs. In the score plot of the first two principal components

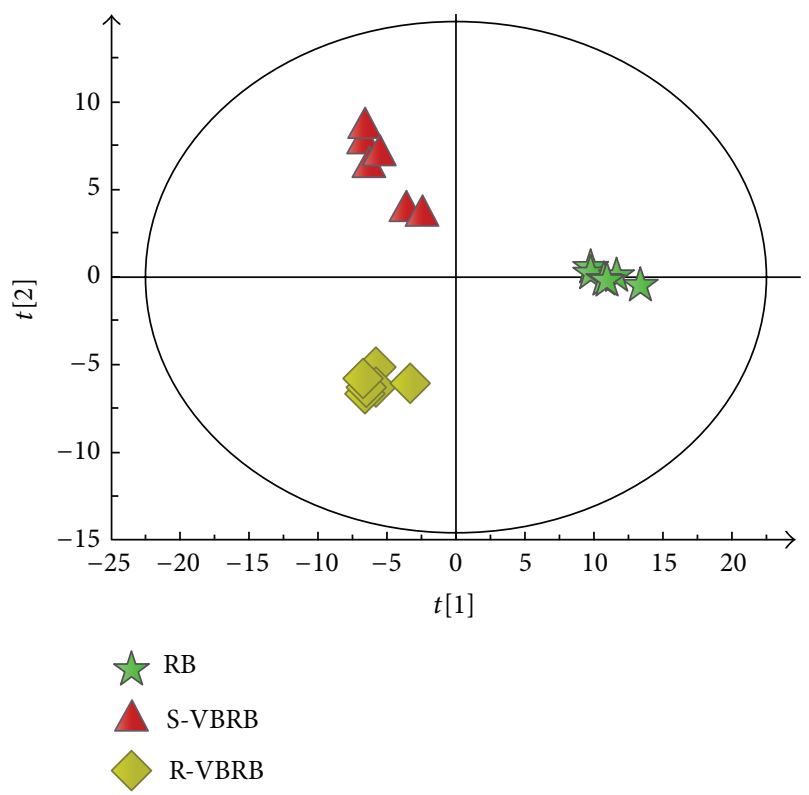

FIgure 2: PCA score plots based on GC-MS data of raw Radix Bupleuri (RB) (pentastar), Shanxi vinegar-baked Radix Bupleuri (S$\mathrm{VBRB}$ ) (triangle), and rice vinegar-baked Radix Bupleuri (R-VBRB) (diamond).

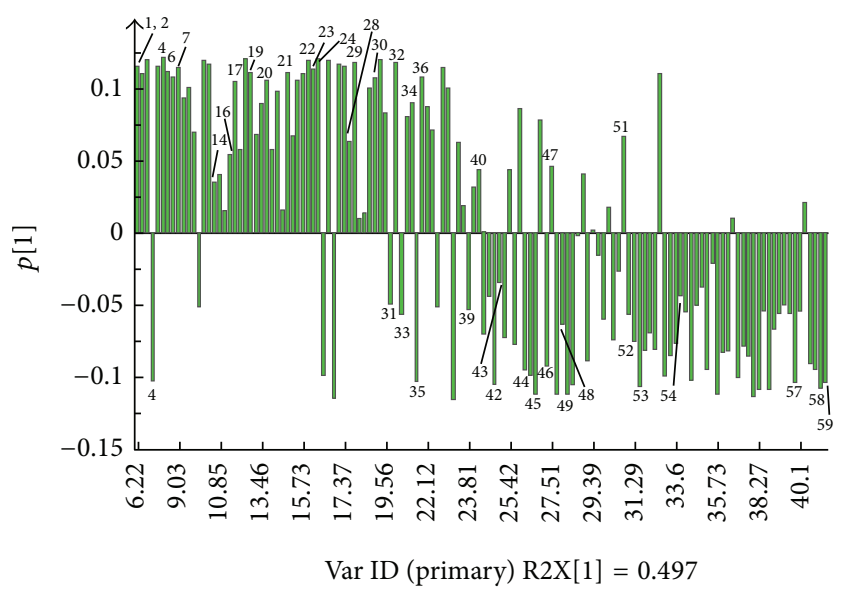

FIGURE 3: Loading (PC1) plot of PCA results obtained from GC-MS spectra.

(PC1: 49.7\%, PC2: 20.4\%), 18 samples were obviously clustered into three groups (Figure 2). The raw RB was located in the positive side of $\mathrm{PCl}$, while the two vinegar-baked RBs were located on the negative side of $\mathrm{PCl}$, which can be further separated by PC2. The separation between the RB and VBRB was more remarkable than those between the two VBRBs, and the observed separation indicated that RB and VBRB were obviously different in their volatile components.

The corresponding loadings plot of $\mathrm{PCl}$ (Figure 3) were used to find the components that are responsible for the separation between RB and VBRB. The signals giving a positive effect in $\mathrm{PCl}$ demonstrated that the corresponding metabolites were higher in RB than those in VBRBs. In contrast, the signals with negative values indicated that 
TABLE 1: Volatile compounds and the relative contents in raw and processed Radix Bupleuri $(n=6)$.

\begin{tabular}{|c|c|c|c|c|c|c|}
\hline \multirow{2}{*}{ No. } & \multirow{2}{*}{$t_{R} / \mathrm{min}$} & \multirow{2}{*}{ Compound } & \multirow{2}{*}{ Formula } & \multicolumn{3}{|c|}{ Relative content $/ \%$} \\
\hline & & & & $\mathrm{RB}$ & S-VBRB & R-VBRB \\
\hline 1 & 6.22 & Pentanal & $\mathrm{C}_{5} \mathrm{H}_{10} \mathrm{O}$ & $0.41 \pm 0.06$ & $0.11 \pm 0.02^{* * *}$ & - \\
\hline 2 & 6.98 & n-Hexanal & $\mathrm{C}_{6} \mathrm{H}_{12} \mathrm{O}$ & $0.55 \pm 0.05$ & $0.18 \pm 0.06^{* * *}$ & $0.26 \pm 0.04^{* * *, \#}$ \\
\hline 3 & 7.44 & $\beta$-Pinene & $\mathrm{C}_{10} \mathrm{H}_{16}$ & $0.34 \pm 0.07$ & - & - \\
\hline 4 & 7.69 & Furfural & $\mathrm{C}_{5} \mathrm{H}_{4} \mathrm{O}_{2}$ & $2.83 \pm 0.38$ & $6.46 \pm 1.36^{* * *}$ & $9.12 \pm 1.06^{* * *, \# \#}$ \\
\hline 5 & 8.18 & 2-Amyl furan & $\mathrm{C}_{9} \mathrm{H}_{14} \mathrm{O}$ & $3.31 \pm 0.17$ & $0.33 \pm 0.02^{* * *}$ & $0.40 \pm 0.02^{* * *, \# \# \#}$ \\
\hline 6 & 8.50 & Dodecanal & $\mathrm{C}_{12} \mathrm{H}_{24} \mathrm{O}$ & $0.46 \pm 0.12$ & $0.18 \pm 0.01^{* *}$ & $0.18 \pm 0.06^{* *}$ \\
\hline 7 & 8.69 & 1-Methyl-2-isopropyl benzene & $\mathrm{C}_{10} \mathrm{H}_{14}$ & $0.81 \pm 0.12$ & $0.46 \pm 0.06^{* * *}$ & $0.52 \pm 0.06^{* * *}$ \\
\hline 8 & 9.03 & 1-Methyl-4-(1-methylethyl)-cyclohexadiene & $\mathrm{C}_{10} \mathrm{H}_{16}$ & $0.21 \pm 0.02$ & - & - \\
\hline 9 & 9.26 & 1-Methyl-4-isopropyl benzene & $\mathrm{C}_{10} \mathrm{H}_{14}$ & $0.71 \pm 0.13$ & $0.36 \pm 0.05^{* *}$ & $0.53 \pm 0.05^{*, \#}$ \\
\hline 10 & 9.48 & 1-Methyl-4-(1-methylethylidene)-cyclohexene & $\mathrm{C}_{10} \mathrm{H}_{16}$ & $0.89 \pm 0.08$ & $0.34 \pm 0.03^{* * *}$ & $0.59 \pm 0.11^{* * *, \# \#}$ \\
\hline 11 & 10.01 & Benzaldehyde & $\mathrm{C}_{7} \mathrm{H}_{6} \mathrm{O}$ & $0.42 \pm 0.04$ & $0.55 \pm 0.03^{* * *}$ & $0.44 \pm 0.08^{\#}$ \\
\hline 12 & 10.14 & 1,2-Cyclooctene oxide & $\mathrm{C}_{8} \mathrm{H}_{14} \mathrm{O}$ & $0.80 \pm 0.16$ & - & - \\
\hline 13 & 10.51 & n-Nonaldehyde & $\mathrm{C}_{9} \mathrm{H}_{18} \mathrm{O}$ & $0.65 \pm 0.09$ & - & - \\
\hline 14 & 10.67 & (E)-2-Octenal & $\mathrm{C}_{8} \mathrm{H}_{14} \mathrm{O}$ & $0.55 \pm 0.12$ & $0.43 \pm 0.07$ & $0.54 \pm 0.13$ \\
\hline 15 & 11.31 & (E)-2,4-Nonadienal & $\mathrm{C}_{9} \mathrm{H}_{14} \mathrm{O}$ & $1.73 \pm 0.07$ & $1.58 \pm 0.11^{*}$ & $1.85 \pm 0.22$ \\
\hline 16 & 11.64 & 7-Methyl-1-nonyl acetylene & $\mathrm{C}_{10} \mathrm{H}_{18}$ & $0.69 \pm 0.04$ & $0.68 \pm 0.07$ & $0.43 \pm 0.02^{*, \#}$ \\
\hline 17 & 12.36 & (E)-9-Tetradecen-1-ol & $\mathrm{C}_{14} \mathrm{H}_{28} \mathrm{O}$ & $2.69 \pm 0.40$ & $2.15 \pm 0.20^{*}$ & $2.37 \pm 0.60$ \\
\hline 18 & 12.61 & Verbenol & $\mathrm{C}_{10} \mathrm{H}_{16} \mathrm{O}$ & $0.79 \pm 0.04$ & - & - \\
\hline 19 & 12.83 & 4-Ethyl-benzenemethanol & $\mathrm{C}_{9} \mathrm{H}_{12} \mathrm{O}$ & $0.83 \pm 0.03$ & $0.30 \pm 0.05^{* * *}$ & $0.51 \pm 0.03^{* * *, \# \# \#}$ \\
\hline 20 & 13.70 & (E,E)-2,4-Sebacic olefin aldehyde & $\mathrm{C}_{10} \mathrm{H}_{16} \mathrm{O}$ & $2.09 \pm 0.15$ & $1.22 \pm 0.16^{* * *}$ & $1.34 \pm 0.21^{* * *}$ \\
\hline 21 & 14.89 & Guaiacol & $\mathrm{C}_{7} \mathrm{H}_{8} \mathrm{O}_{2}$ & $1.31 \pm 0.09$ & $0.74 \pm 0.07^{* * *}$ & $0.37 \pm 0.05^{* * *, \# \# \#}$ \\
\hline 22 & 15.73 & 2-(1,1-Dimethylethyl)-phenol & $\mathrm{C}_{10} \mathrm{H}_{14} \mathrm{O}$ & $0.79 \pm 0.05$ & $0.56 \pm 0.05^{* * *}$ & $0.50 \pm 0.10^{* * *}$ \\
\hline 23 & 15.90 & 1-(1-Cyclohexen-1-yl)-ethanone & $\mathrm{C}_{8} \mathrm{H}_{12} \mathrm{O}$ & $1.00 \pm 0.09$ & $0.52 \pm 0.06^{* * *}$ & $0.51 \pm 0.04^{* * *}$ \\
\hline 24 & 16.41 & 1-Isopropyl-2-methoxy-4-toluene & $\mathrm{C}_{11} \mathrm{H}_{16} \mathrm{O}$ & $3.19 \pm 0.31$ & $0.74 \pm 0.06^{* * *}$ & $0.52 \pm 0.11^{* * *, \# \#}$ \\
\hline 25 & 16.63 & 2,4-Dimethoxytoluene & $\mathrm{C}_{9} \mathrm{H}_{12} \mathrm{O}_{2}$ & - & $0.67 \pm 0.13$ & $0.91 \pm 0.23$ \\
\hline 26 & 16.78 & 2-Decenal & $\mathrm{C}_{10} \mathrm{H}_{12} \mathrm{O}_{2}$ & $0.4 \pm 0.08$ & - & - \\
\hline 27 & 17.07 & 2-Nonyl acetylene & $\mathrm{C}_{9} \mathrm{H}_{16}$ & - & $0.68 \pm 0.09$ & $0.62 \pm 0.10$ \\
\hline 28 & 17.73 & (Z)-2-Decenal & $\mathrm{C}_{10} \mathrm{H}_{18} \mathrm{O}$ & $1.07 \pm 0.15$ & $0.96 \pm 0.05$ & $0.82 \pm 0.19^{*}$ \\
\hline 29 & 18.26 & p-Ethyl guaiacol & $\mathrm{C}_{9} \mathrm{H}_{12} \mathrm{O}_{2}$ & $1.73 \pm 0.11$ & $0.95 \pm 0.21^{* * *}$ & $0.82 \pm 0.10^{* * *}$ \\
\hline 30 & 19.09 & Capraldehyde & $\mathrm{C}_{10} \mathrm{H}_{20} \mathrm{O}$ & $2.24 \pm 0.13$ & $1.41 \pm 0.29^{* * *}$ & $1.46 \pm 0.21^{* *}$ \\
\hline 31 & 19.87 & (Z)-9-Hexadecen-1-ol & $\mathrm{C}_{16} \mathrm{H}_{32} \mathrm{O}$ & $1.16 \pm 0.09$ & $1.37 \pm 0.28$ & $1.39 \pm 0.14^{*}$ \\
\hline 32 & 20.04 & 4-Dimethyl-3-cyclohexene-1-acetaldehyde & $\mathrm{C}_{10} \mathrm{H}_{16} \mathrm{O}$ & $6.02 \pm 0.77$ & $2.01 \pm 0.38^{* * *}$ & $2.46 \pm 0.36^{* * *}$ \\
\hline 33 & 20.93 & Eucalyptol & $\mathrm{C}_{10} \mathrm{H}_{18} \mathrm{O}$ & $0.60 \pm 0.02$ & $0.72 \pm 0.06^{* *}$ & $0.66 \pm 0.10$ \\
\hline 34 & 21.12 & Menthol & $\mathrm{C}_{10} \mathrm{H}_{20} \mathrm{O}$ & $0.50 \pm 0.04$ & $0.32 \pm 0.06^{* * *}$ & $0.43 \pm 0.06^{*, \#}$ \\
\hline 35 & 21.73 & 6-Heptyltetrahydro-2H-pyran-2-one & $\mathrm{C}_{12} \mathrm{H}_{22} \mathrm{O}_{2}$ & $0.48 \pm 0.07$ & $0.68 \pm 0.06^{* * *}$ & $0.89 \pm 0.08^{* * *, \# \# \#}$ \\
\hline 36 & 21.92 & 2-Methoxy-4-propyl-phenol & $\mathrm{C}_{10} \mathrm{H}_{14} \mathrm{O}_{2}$ & $0.86 \pm 0.14$ & $0.43 \pm 0.05^{* * *}$ & $0.28 \pm 0.05^{* * *, \# \#}$ \\
\hline 37 & 23.08 & $\alpha$-Cubebene & $\mathrm{C}_{15} \mathrm{H}_{24}$ & - & $0.97 \pm 0.19$ & $1.13 \pm 0.10$ \\
\hline 38 & 23.42 & Methychavicol & $\mathrm{C}_{10} \mathrm{H}_{12} \mathrm{O}$ & $1.31 \pm 0.18$ & $1.49 \pm 0.08$ & 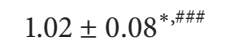 \\
\hline 39 & 23.81 & Thymol & $\mathrm{C}_{10} \mathrm{H}_{14} \mathrm{O}$ & $8.05 \pm 0.44$ & $8.63 \pm 0.42^{*}$ & $8.37 \pm 0.18$ \\
\hline 40 & 24.23 & (6E)-6-Tridecen-4-yne & $\mathrm{C}_{13} \mathrm{H}_{22}$ & $1.36 \pm 0.16$ & $1.22 \pm 0.13$ & $1.27 \pm 0.17$ \\
\hline 41 & 24.45 & 6-Tert-butyl-2,4-dimethylphenol & $\mathrm{C}_{12} \mathrm{H}_{18} \mathrm{O}$ & $4.12 \pm 0.49$ & $4.86 \pm 0.23^{*}$ & $4.54 \pm 0.21^{\#}$ \\
\hline 42 & 24.89 & 1,3-Bis(1-methylethyl)-benzene & $\mathrm{C}_{12} \mathrm{H}_{18}$ & $0.53 \pm 0.07$ & $1.36 \pm 0.26^{* * *}$ & $1.22 \pm 0.12^{* * *}$ \\
\hline 43 & 25.14 & 2-Methyl-5-(1-methylethyl)-phenol & $\mathrm{C}_{10} \mathrm{H}_{14} \mathrm{O}$ & $1.07 \pm 0.05$ & $1.22 \pm 0.15$ & $1.07 \pm 0.11$ \\
\hline 44 & 26.10 & 1,2-Dimethoxy-4-(1-propenyl)-benzene & $\mathrm{C}_{11} \mathrm{H}_{14} \mathrm{O}_{2}$ & $1.14 \pm 0.35$ & $1.81 \pm 0.17^{* *}$ & $2.39 \pm 0.27^{* * *, \# \#}$ \\
\hline 45 & 26.73 & $\alpha$-Ylangene & $\mathrm{C}_{15} \mathrm{H}_{24}$ & $0.46 \pm 0.04$ & $1.35 \pm 0.22^{* * *}$ & $1.66 \pm 0.30^{* * *}$ \\
\hline 46 & 27.23 & $\alpha$-Guaiene & $\mathrm{C}_{15} \mathrm{H}_{24}$ & $0.89 \pm 0.10$ & $1.83 \pm 0.39^{* *}$ & $1.76 \pm 0.36^{* *}$ \\
\hline 47 & 27.51 & 1,2-Dihydro-acenaphthene & $\mathrm{C}_{12} \mathrm{H}_{10}$ & $3.05 \pm 0.42$ & $3.29 \pm 0.59$ & $2.58 \pm 0.30^{*, \#}$ \\
\hline 48 & 28.01 & $\beta$-Ylangene & $\mathrm{C}_{15} \mathrm{H}_{24}$ & $2.32 \pm 0.26$ & $2.58 \pm 0.44$ & $2.95 \pm 0.18^{* *}$ \\
\hline
\end{tabular}


TABLe 1: Continued.

\begin{tabular}{|c|c|c|c|c|c|c|}
\hline \multirow{2}{*}{ No. } & \multirow{2}{*}{$t_{R} / \min$} & \multirow{2}{*}{ Compound } & \multirow{2}{*}{ Formula } & \multicolumn{3}{|c|}{ Relative content/\% } \\
\hline & & & & $\mathrm{RB}$ & S-VBRB & R-VBRB \\
\hline 49 & 28.28 & Isoledene & $\mathrm{C}_{15} \mathrm{H}_{24}$ & $0.68 \pm 0.06$ & $1.60 \pm 0.26^{* * *}$ & $1.49 \pm 0.26^{* * *}$ \\
\hline 50 & 29.96 & 1,6,7-Trimethylnaphthalene & $\mathrm{C}_{13} \mathrm{H}_{14}$ & $0.68 \pm 0.04$ & $0.92 \pm 0.33$ & $0.56 \pm 0.14^{\#}$ \\
\hline 51 & 30.86 & Palmitic acid & $\mathrm{C}_{16} \mathrm{H}_{32} \mathrm{O}_{2}$ & $3.85 \pm 0.57$ & $2.27 \pm 0.47^{* * *}$ & $3.17 \pm 0.39^{\#}$ \\
\hline 52 & 31.29 & Caryophyllene oxide & $\mathrm{C}_{15} \mathrm{H}_{24} \mathrm{O}$ & $0.88 \pm 0.12$ & $1.32 \pm 0.37^{*}$ & $1.36 \pm 0.23^{*}$ \\
\hline 53 & 31.60 & 9-Octadecenoate methyl & $\mathrm{C}_{19} \mathrm{H}_{36} \mathrm{O}_{2}$ & $0.49 \pm 0.06$ & $0.90 \pm 0.13^{* * *}$ & $0.90 \pm 0.15^{* * *}$ \\
\hline 54 & 33.86 & Moslene & $\mathrm{C}_{10} \mathrm{H}_{16}$ & $0.97 \pm 0.12$ & $0.98 \pm 0.05$ & $2.96 \pm 0.51^{* * *, \# \# \#}$ \\
\hline 55 & 35.73 & 6-Methyl-2-(4-methylphenyl)-5-heptylene & $\mathrm{C}_{15} \mathrm{H}_{22}$ & - & $0.18 \pm 0.03$ & $0.20 \pm 0.02$ \\
\hline 56 & 38.15 & Nerolidol & $\mathrm{C}_{15} \mathrm{H}_{26} \mathrm{O}$ & - & $0.45 \pm 0.09$ & $0.44 \pm 0.10$ \\
\hline 57 & 39.87 & Methyl palmitate & $\mathrm{C}_{17} \mathrm{H}_{34} \mathrm{O}_{2}$ & $0.19 \pm 0.05$ & $1.14 \pm 0.31^{* * *}$ & $0.96 \pm 0.23^{* * *}$ \\
\hline 58 & 40.49 & Stearic acid & $\mathrm{C}_{18} \mathrm{H}_{36} \mathrm{O}_{2}$ & $0.05 \pm 0.03$ & $1.02 \pm 0.43^{* * *}$ & $0.49 \pm 0.08^{* * *, \#}$ \\
\hline 59 & 41.15 & Linoleic acid & $\mathrm{C}_{18} \mathrm{H}_{32} \mathrm{O}_{2}$ & $0.13 \pm 0.05$ & $1.07 \pm 0.20^{* * *}$ & $0.83 \pm 0.13^{* *}$ \\
\hline
\end{tabular}

* means compared to RB $\left({ }^{*} p<0.05,{ }^{* *} p<0.01,{ }^{* * *} p<0.001\right)$; \# means compared to S-VBRB $\left({ }^{\#} p<0.05,{ }^{\# \#} p<0.01,{ }^{\# \# \#} p<0.001\right)$.

the level of related components was higher in VBRBs. The signals of pentanal, n-hexanal, 2-amyl furan, dodecanal, 1methyl-2-isopropyl benzene, 1-methyl-4-isopropyl benzene, 1-methyl-4-(1-methylethylidene)-cyclohexene, (E)-2-octenal, 7-methyl-1-nonyl acetylene, (E)-9-tetradecen-1-ol, 4-ethylbenzenemethanol, (E,E)-2,4-sebacic olefin aldehyde, guaiacol, 2-(1,1-dimethylethyl)-phenol, 1-(1-cyclohexen-1-yl)ethanone, 1-isopropyl-2-methoxy-4-toluene, (Z)-2-decenal, p-ethyl guaiacol, capraldehyde, 4-dimethyl-3-cyclohexene-1acetaldehyde, menthol, 2-methoxy-4-propyl-phenol, (6E)-6tridecen-4-yne, 1,2-dihydro-acenaphthene, and palmitic acid gave a positive contribution to PCl. The signals with negative values in PC1 included furfural, Z-9-hexadecen-1-ol, eucalyptol, 6-heptyltetrahydro-2H-pyran-2-one, thymol, 6-tert-butyl-2,4-dimethylphenol, 1,3-bis(1-methylethyl)benzene, 2-methyl-5-(1-methylethyl)-phenol, 1,2-dimethoxy4-(1-propenyl)-benzene, $\alpha$-ylangene, $\alpha$-guaiene, $\beta$-ylangene, isoledene, caryophyllene oxide, 9-octadecenoate methyl, moslene, methyl palmitate, stearic acid, and linoleic acid.

The two VBRBs could be clearly separated by PC2. The S-VBRB was grouped on the positive side of PC2, while the R-VBRB was on the negative side of PC2. The corresponding loadings plot (Figure 4) clearly showed that high levels of pentanal, benzaldehyde, 7-methyl-1nonyl acetylene, guaiacol, 2-(1,1-dimethylethyl)-phenol, (Z)-2-decenal, eucalyptol, 2-methoxy-4-propyl-phenol, methychavicol, 6-tert-butyl-2,4-dimethylphenol, 2-methyl-5-(1methylethyl)-phenol, $\alpha$-guaiene, 1,2-dihydro-acenaphthene, 1,6,7-trimethylnaphthalene, methyl palmitate, stearic acid, and linoleic acid were present in the S-VBRB. In contrast, higher amounts of n-hexanal, furfural, 1-methyl-2-isopropyl benzene, 1-methyl-4-isopropyl benzene, 1-methyl-4-(1-methylethylidene)-cyclohexene, (E)-2-octenal, (E)-2,4-nonyl diene ether, (E)-9-tetradecen-1-ol, 4-ethyl-benzenemethanol, (E,E)-2,4-sebacic olefin aldehyde, 2,4-dimethoxytoluene, (Z)-9-hexadecen-1-ol, menthol, 6-heptyltetrahydro-2Hpyran-2-one, (6E)-6-tridecen-4-yne, 1,2-dimethoxy-4-(1propenyl)-benzene, $\alpha$-ylangene, $\beta$-ylangene, palmitic acid, moslene, and 6-methyl-2-(4-methylphenyl)-5-heptylene were present in R-VBRB.

Table 1 showed the relative contents of identified volatile components in raw and processed RBs, and all the results

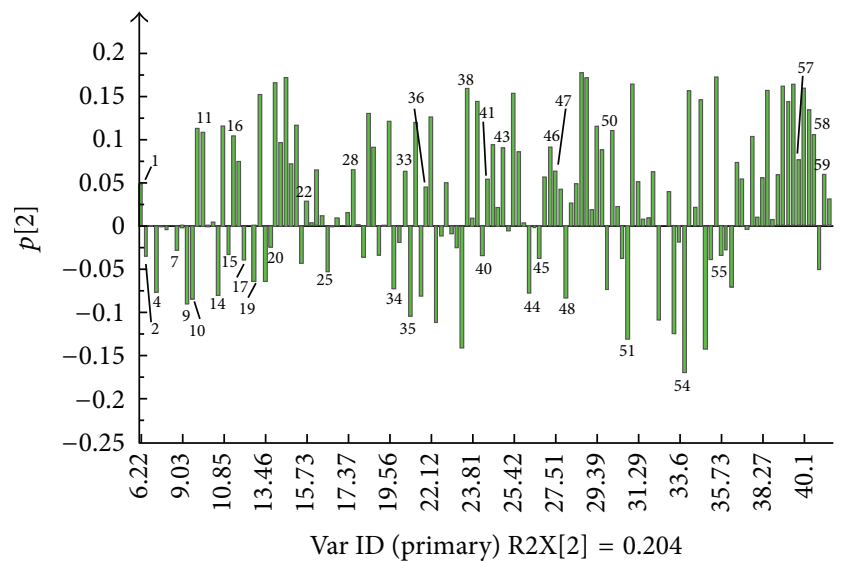

FIGURE 4: Loading (PC2) plot of PCA results obtained from GC-MS spectra.

were shown as mean \pm SD. Statistical analysis was carried out using one-way ANOVA by SPSS. The results obtained by quantitative statistical analysis were in agreement with those of multivariate analysis.

To visualize the changes between the raw and two processed RBs, heat map was further generated based on the differential compounds determined above (Figure 5). Here, the red and green colors corresponded to increased and decreased constituents in the VBRB after processing, respectively. It was obvious from the left side that the 18 samples of RB and VBRB could be clearly divided into two main clusters, and S-VBRB and R-VBVB could be separated in the second cluster. The compounds could be also divided into two main clusters on the top, and the left cluster that presented these ingredients were rich in raw $\mathrm{RB}$, while the compounds in the right cluster showed high contents in VBRB.

\section{Conclusion}

In the present study, GC-MS coupled with multivariate data analysis was applied to compare the volatile components between crude and two vinegar-processed RBs. Compared 


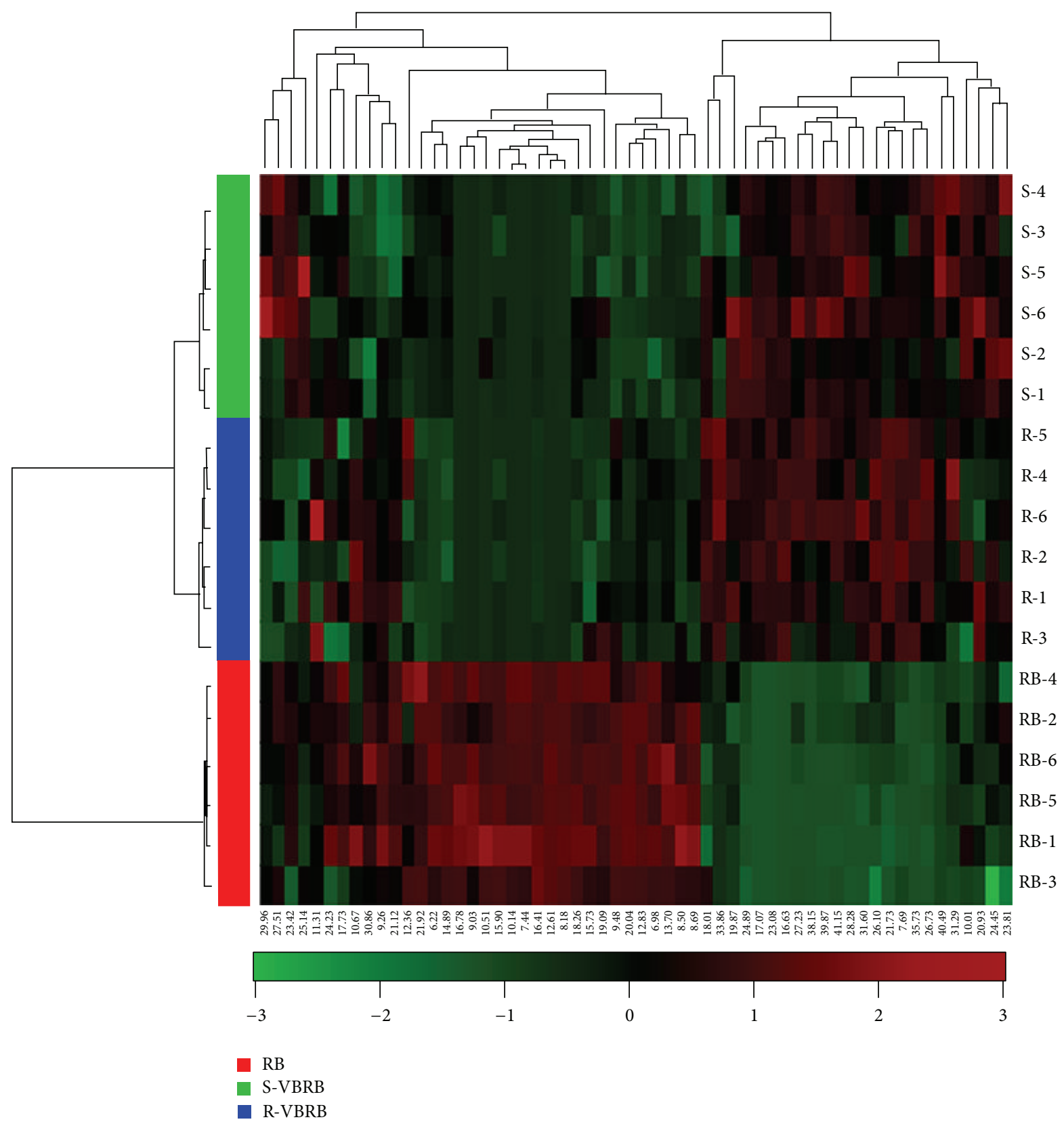

FIgURE 5: Heat maps of differential components between raw and processed Radix Bupleuri.

with the previous studies, more chemical components were identified, and the influence of vinegar type on processing was also discussed.

After vinegar-baking, not only the oil yields were decreased significantly, but the chemical compositions were also changed, including the disappearance or appearance, as well as the content increase or decrease of some volatile compounds. According to the theory of TCM, the purpose of herb processing is to increase potency, reduce toxicity and side effects, and alter the properties or functions [27]. The relationship between the change of volatile oil in $\mathrm{RB}$ and the drug action should be further investigated.

Two different VBRBs also showed differences in the volatile compositions, suggesting that the type of vinegar exerted great impacts on the vinegar-baking process. Thus, in order to ensure the therapeutic effect and safety of VBRB in clinic, the effect of processing by different vinegars should be further investigated on the other herbal drugs.

\section{Conflict of Interests}

All authors declare no conflict of interests.

\section{Acknowledgments}

This study was financially supported by the Program for the Outstanding Innovative Teams of Higher Learning 
Institutions of Shanxi (OIT), Program of Science and Technology of Shanxi Province (no. 20140311023-4), and Science and Technology Innovation Team of Shanxi Province (no. 2013131015).

\section{References}

[1] Chinese Pharmacopoeia Commission, The Pharmacopoeia of the People's Republic of China, Vol. I: Chinese Materia Medica, Chemical Industry Press, Beijing, China, 2010.

[2] M. K. Seo, H. Y. Cho, C. H. Lee et al., "Antioxidant and proliferative activities of bupleuri radix extract against serum deprivation in SH-SY5Y cells," Psychiatry Investigation, vol. 10, no. 1, pp. 81-88, 2013.

[3] X. Q. Cheng, L. J. Song, H. Li, H. Di, Y. Y. Zhang, and D. F. Chen, "Beneficial effect of the polysaccharides from bupleurum smithii var. parvifolium on 'two-Hit' acute lung injury in rats," Inflammation, vol. 35, no. 5, pp. 1715-1722, 2012.

[4] D. Q. Wang, X. W. Li, F. S. Zhang, J. Xing, Z. Y. Li, and X. M. Qin, "Antipyretic effect of Bupleurum scorzonerifolium based on GC-MS metabonomics," Chinese Traditional and Herbal Drugs, vol. 44, no. 5, pp. 574-580, 2013.

[5] J. Lee, D. H. Yang, J. H. Suh et al., "Species discrimination of Radix Bupleuri through the simultaneous determination of ten saikosaponins by high performance liquid chromatography with evaporative light scattering detection and electrospray ionization mass spectrometry," Journal of Chromatography B, vol. 879, no. 32, pp. 3887-3895, 2011.

[6] H. Q. Huang, J. Su, X. Zhang, L. Shan, and W. D. Zhang, "Qualitative and quantitative determination of polyacetylenes in different Bupleurum species by high performance liquid chromatography with diode array detector and mass spectrometry," Journal of Chromatography A, vol. 1218, no. 8, pp. 1131-1138, 2011.

[7] Z. L. Wang and X. B. Wang, "Traditional Chinese medicine processing principle and its value analysis in clinic," China Journal of Chinese Medicine, vol. 29, no. 188, pp. 86-87, 2014.

[8] C. J. Seneviratne, R. W. K. Wong, and L. P. Samaranayake, "Potent anti-microbial activity of traditional Chinese medicine herbs against Candida species," Mycoses, vol. 51, no. 1, pp. 30-34, 2008.

[9] Y. Cheng, Y. Huang, Y. Tian, L. Xu, G. Q. Liu, and Z. J. Zahng, "Assessment of the effects of Radix Bupleuri and vinegarbaked Radix Bupleuri on cytochrome 450 activity by a six-drug cocktail approach," Chinese Journal of Natural Medicines, vol. 11, no. 3, pp. 302-308, 2013.

[10] R. Z. Zhao, D. Yuan, S. J. Liu, Y. J. Chen, L. J. Liu, and Y. Zhao, "Liver targeting effect of vinegar-baked Radix Bupleuri on rhein in rats," Journal of Ethnopharmacology, vol. 132, no. 2, pp. 421428, 2010.

[11] X. Chen, T. Yu, Z. Chen, R. Zhao, and S. Mao, "Effect of saikosaponins and extracts of vinegar-baked Bupleuri Radix on the activity of $\beta$-glucuronidase," Xenobiotica, vol. 44, no. 9, pp. 785-791, 2014.

[12] L. Xu, J. X. Tian, R. Song, G. Q. Liu, Y. Tian, and Z. J. Zhang, "LC-MS/MS determination and comparison of saikosaponin $\mathrm{a}, \mathrm{b}_{2}, \mathrm{c}, \mathrm{d}$ in crude and processed radix bupleuri by vinegar," Journal of China Pharmaceutical University, vol. 43, no. 4, pp. 334-340, 2012.

[13] H. Jiang, J. Li, R. B. Shi, and W. P. Yin, "Influence of processing on four saikosaponins in Radix Bupleuri," Chinese Pharmaceutical Journal, vol. 44, no. 21, pp. 1618-1621, 2009.
[14] D. H. Xie, X. B. Jia, B. C. Cai, and L. S. Zhang, "Experimental study on anti-inflammatory and analgesic effects of volatile oil of Bupleurum Chinense and B. scorzonerifolium," Pharmaceutical and Clinical Research, vol. 15, no. 2, pp. 108-110, 2007.

[15] Y. Xue and J. Y. Bai, "Compatative study of the antipyretic composition in Radix Bupleuri," Pharmacology and Clinics of Chinese Materia Medica, vol. 19, no. 1, pp. 11-12, 2003.

[16] X. D. Li, "Comparative analysis of active compositions of Radix Bupleuri before and after being processed," Chinese Traditional Patent Medicine, vol. 22, no. 7, pp. 483-485, 2000.

[17] C. M. Yin, L. Li, and Y. J. Wang, "Components research of Radix Bupleurum and it processed products," Liaoning Pharmacy and Clinical Rehedies, vol. 4, no. 4, p. 176, 2001.

[18] Z. L. Bai, Y. Wang, and T. Z. Jia, "GC-MS analysis of volatile oil of Radix Bupleuri before and after being processed," Chinese Traditional Patent Medicine, vol. 31, no. 9, pp. 1397-1398, 2009.

[19] J. Lisec, N. Schauer, J. Kopka, L. Willmitzer, and A. R. Fernie, "Gas chromatography mass spectrometry-based metabolite profiling in plants," Nature Protocols, vol. 1, no. 1, pp. 387-396, 2006.

[20] J. Maree, G. Kamatou, S. Gibbons, A. Viljoen, and S. Van Vuuren, "The application of GC-MS combined with chemometrics for the identification of antimicrobial compounds from selected commercial essential oils," Chemometrics and Intelligent Laboratory Systems, vol. 130, pp. 172-181, 2014.

[21] X. Gao, M. Xie, S. Liu et al., "Chromatographic fingerprint analysis of metabolites in natural and artificial agarwood using gas chromatography-mass spectrometry combined with chemometric methods," Journal of Chromatography B, vol. 967, pp. 264-273, 2014.

[22] X. Deng, Q. Liao, X. Xu et al., "Analysis of essential oils from cassia bark and cassia twig samples by GC-MS combined with multivariate data analysis," Food Analytical Methods, vol. 7, no. 9, pp. 1840-1847, 2014.

[23] I. H. Cho, H. J. Lee, and Y. S. Kim, "Differences in the volatile compositions of ginseng species (Panax sp.)," Journal of Agricultural and Food Chemistry, vol. 60, no. 31, pp. 7616-7622, 2012.

[24] G. Cao, H. Cai, J. Jiang et al., "Chemical differentiation of volatile compounds in crude and processed Atractylodis Macrocephalae Rhizoma by using comprehensive twodimensional gas chromatography with time-of-flight mass spectrometry combined with multivariate data analysis," Journal of Separation Science, vol. 37, no. 9-10, pp. 1194-1198, 2014.

[25] S. H. Liu, S. S. Lu, Y. L. Su, and Y. Guo, "Analysis of volatile compounds in Radix bupleuri injection by GC-MS-MS," Chromatographia, vol. 74, no. 5-6, pp. 497-502, 2011.

[26] X. Li, Y. Jia, A. Song, X. Chen, and K. Bi, "Analysis of the essential oil from Radix Bupleuri using capillary gas chromatography," Yakugaku Zasshi, vol. 125, no. 10, pp. 815-819, 2005.

[27] B. Tang, J. Ding, Y. Yang, F. Wu, and F. Song, "Systems biochemical responses of rats to Kansui and vinegar-processed Kansui exposure by integrated metabonomics," Journal of Ethnopharmacology, vol. 153, no. 2, pp. 511-520, 2014. 


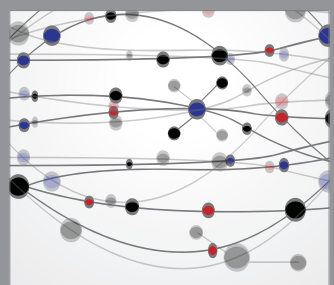

The Scientific World Journal
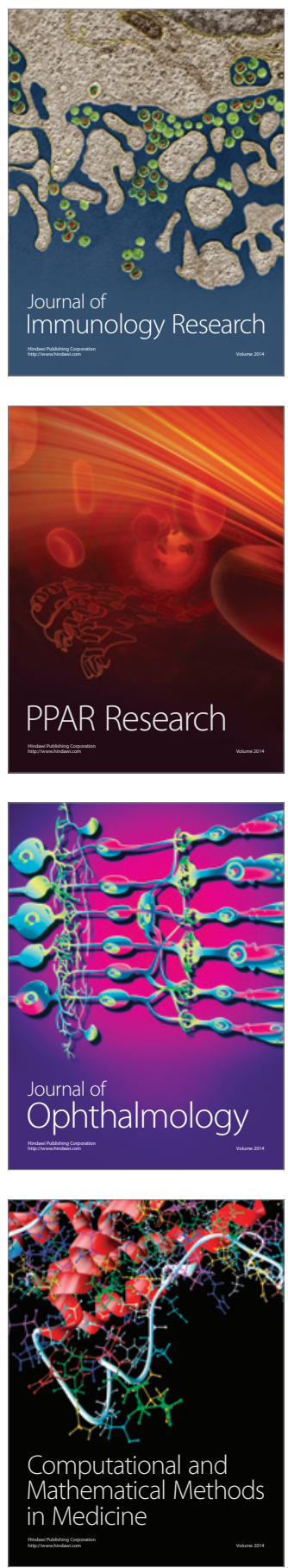

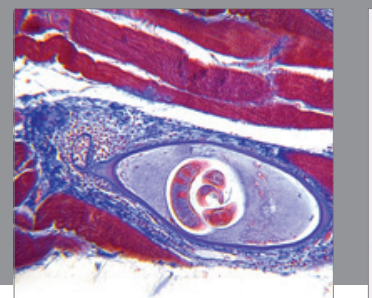

Gastroenterology

Research and Practice
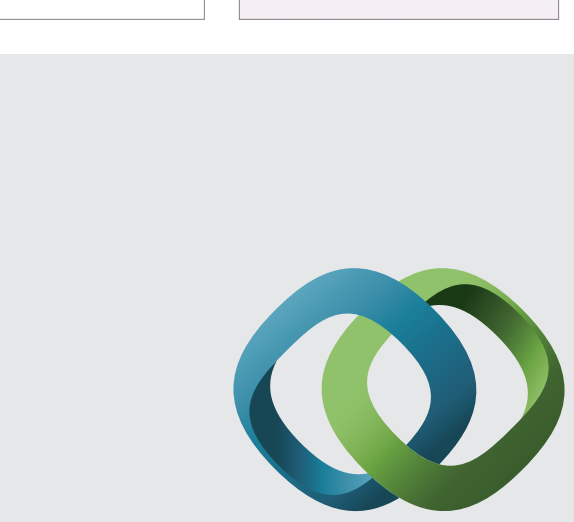

\section{Hindawi}

Submit your manuscripts at

http://www.hindawi.com
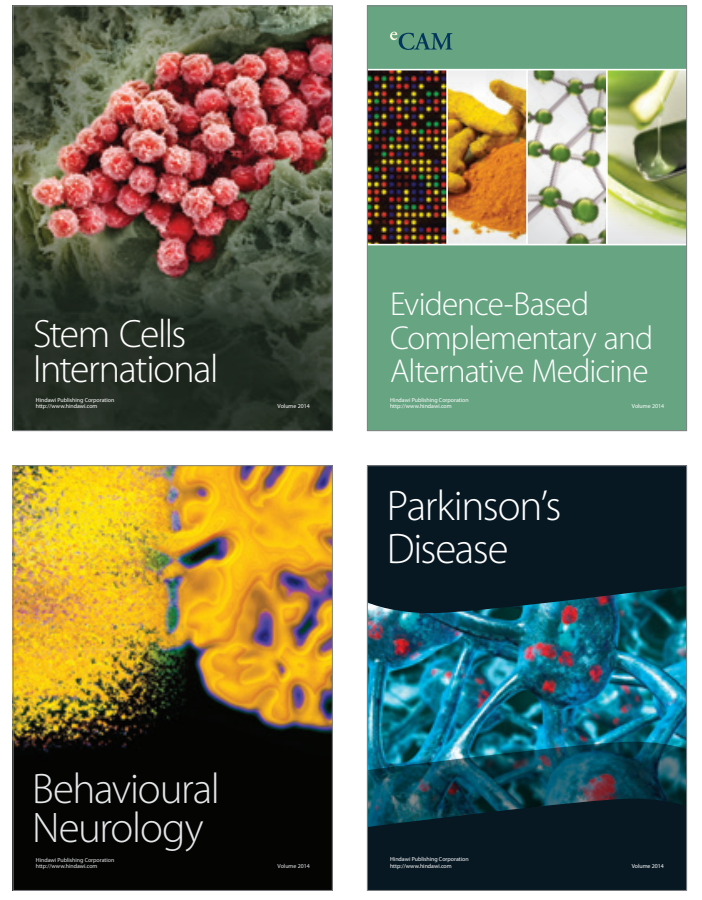
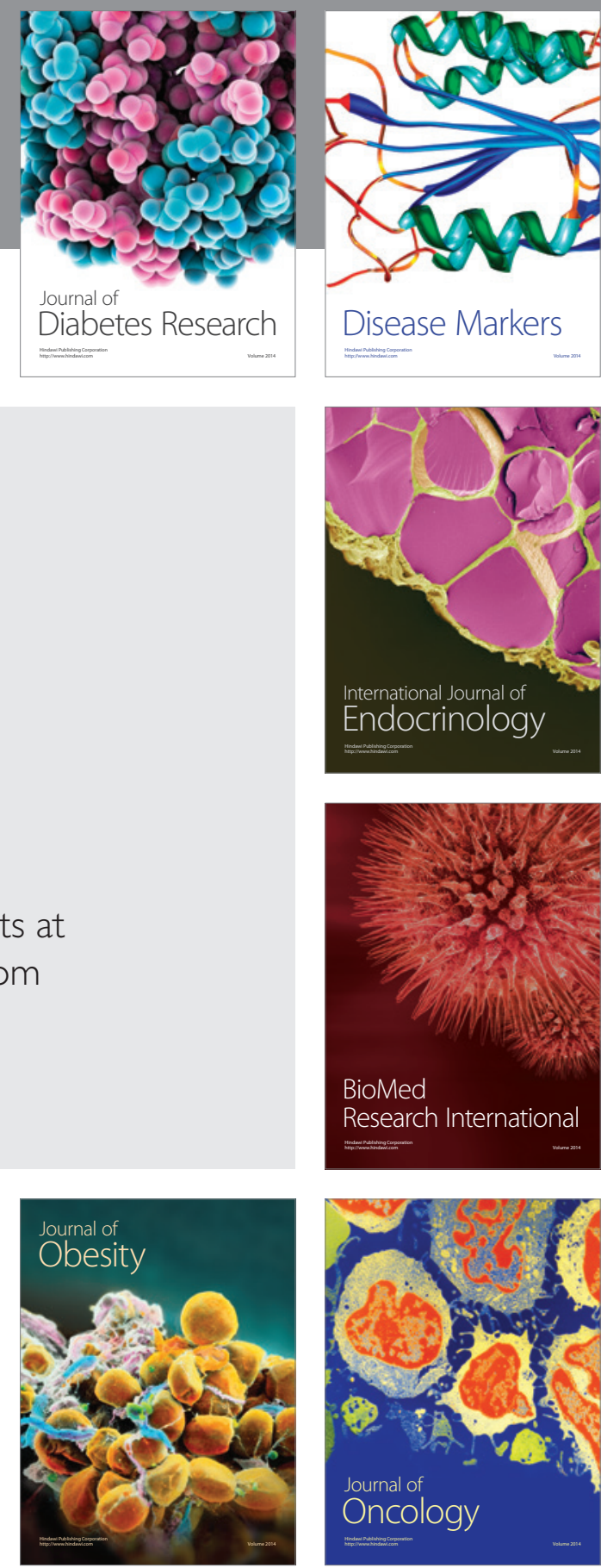

Disease Markers
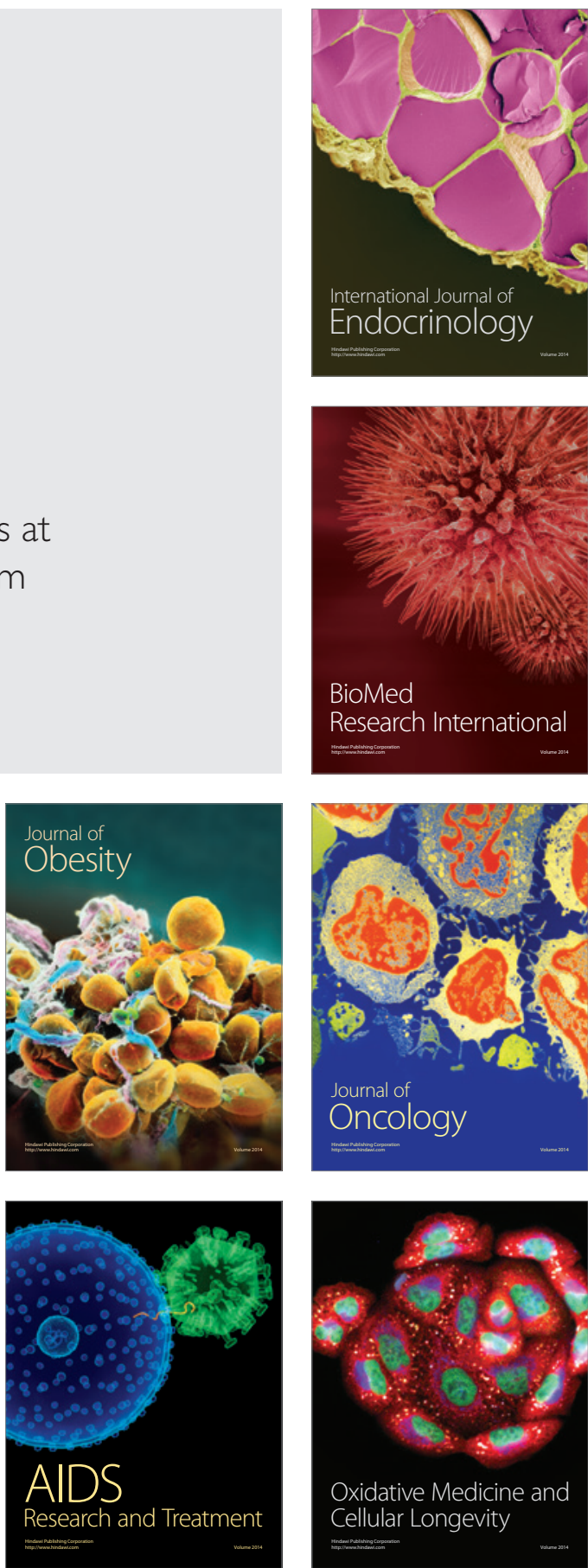